\title{
Drug Residues in Foods of Animal Origin and Their Impact on Human Health: Review
}

\author{
Tamirat Herago* \\ Ethiochicken, AGP Poultry PLC, Hawassa, Ethiopia \\ Abebe Agonafir \\ Jigjiga Univeristy, college of Veterinary Medicine, Jigjiga, Ethiopia \\ Tamirat Chuko \\ Humbo Woreda Livestock and Fishery office, Wolaita Zone, Ethiopia
}

\begin{abstract}
The safety of human food is threatened by various agents including pathogenic microorganisms, and antimicrobial agents. Antibiotics are natural products of a micro-organism, or similar semi-synthetic products that inhibit the growth or destroy microorganisms. Residues of veterinary medicines are defined as pharmacologically active substances, principles, or degradation products and their metabolites, which remain in foodstuffs obtained from animals that have been administered the veterinary medicine. The most common causes for the presence of antibiotic residues in food of animal origin are violation of withdrawal periods, overdosing of antibiotics and use of inaccurate routes of administration of drugs. Drug residues concentrations vary from tissue to tissue and are generally observed to be higher in tissues of storage such as body fat or in organs that actively metabolize and excrete them. Probably the most common way milk is contaminated by penicillin is through intra-mammary infusion of the drug in the treatment of mastitis. The presence of antibiotics or their metabolites in food is potentially hazardous to health as it may cause allergic reactions in people and antibiotic resistance in pathogenic microorganisms. Some antibiotics like streptomycin, neomycin and gentamicin has been reported to cause damage to the kidney and to hearing. Allergic reaction to the human body mostly resulted from $\beta$-Lactam antibiotics. Anaphylactic reactions are reported to result from consumption of beef or pork containing penicillin. Drugs in which their residues induce cancer in humans or consumers are: nitrofurans, nitroimdazoles, etc. Antibiotics are invariably administered to dairy cattle, but their indiscriminate use, without adequate technical and veterinary control, can lead to a series of negative consequences at all levels of the dairy productive chain. Many antibacterial and anticoccidial drugs are licensed for use in poultry for treatment of enteric and respiratory disease. Potential adverse effects of drugs that appear as residues in food animals are: carcinogenic, allergic, toxic, neurologic disorder and microbiological effects.
\end{abstract}

Keywords: Drugs, Drug residue, Food, Human

DOI: $10.7176 / \mathrm{FSQM} / 108-02$

Publication date:May $31^{\text {st }} 2021$

\section{Introduction}

The safety of human food is threatened by various agents including pathogenic microorganisms, aflatoxins, pesticides and antimicrobial agents. Pathogenic microorganisms constitute the most important food related threat to public health. Relatively, little is known about food safety in relation to antimicrobial agents, in the developing world. While pasteurization and other forms of heat treatment eliminate pathogenic microorganisms from animal source food, these procedures have limited or variable effects on drug residues in animal originated food (Moat, 1988).

Antibiotics are natural products of a micro-organism, identical synthetic products, or similar semi-synthetic products that inhibit the growth or destroy microorganisms. Due to their availability and low cost, antibiotics have been widely used in the dairy, beef, poultry, aquaculture, and honey production industries in various countries. In veterinary medicine, antibiotics are used for therapeutic, prophylactic, nutritive and growth purposes (Wassenaar, 2005).Antimicrobials are also needed for the relief of pain and suffering in animals. For food animals, the gains that have been made in food production capacity would not have been possible without the ability for reliable drugs to contain the threat of disease to animals (Jones and Seymour, 1988).

In recent years, concerns about the use of antimicrobial products in food producing animals have focused on human food safety because foods of animal origin are sometimes identified as the vehicles of food borne disease in humans and therefore, also vehicles of resistant food borne pathogens and resistant genetic material. The major zoonotic pathogens of concern for the development of antimicrobial resistance are Salmonella species and Campylobacter Jejune(Smith et al., 2004).

The benefit to human health in the proper use of antibiotics in food animals is related to the ability for drugs to combat infectious bacteria that can be transferred to humans by either direct contact with the sick animal, 
consumption of food contaminated with pathogens from animals or proliferation into the environment. The use of drugs in animals is fundamental to animal health and well-being. However, the use of antimicrobials in food animals is not without risks (Lee et al., 2005).

Various antibiotics used in the treatment of animal diseases have been shown to occur in animal products used as human food and are usually attributed to non-observance of withdrawal periods before sale of animal source food (Redundant and Moreitain, 1990). Residues of veterinary medicines are defined as pharmacologically active substances whether active principles, or degradation products and their metabolites, which remain in foodstuffs obtained from animals that have been administered the veterinary medicine. The most common causes for the presence of antibiotic residues in food of animal origin are violation of withdrawal periods, overdosing of antibiotics, and use of banned drugs for treatment and injection of drugs through incorrect routes for treatment of economic animals (Lee et al., 2005).

The use of antibiotics in animal husbandry and its occurrence in related food may lead to selection of resistance in bacterial populations that do not respond to treatment commonly used for human diseases. Residues are also concern due to their possible adverse effects on inhibition of starter cultures used to produce cultured milk products such as yogurt and cheeses (Jones and Seymour, 1988).

Though the use of antimicrobials in food animals and their residue in human consuming foods of animals' origin need great concentration, so much works was not conducted to create awareness and emphasis them. Therefore, objectives of this paper were to review the major classes of drugs used in food animals, its residues in animal originated food and to understand the potential adverse effects of drugs residues in human health.

\section{Major classes of drugs used in food animals}

The aim of anti-microbiological therapy is to rapidly produce and maintain an effective concentration of drug at the site of infection for sufficient time to allow host specific and non-specific defences to eradicate the pathogen (Benet and Belleemain, 2005).

There are many drugs that used in food producing animals. Some of them are: topical antiseptics, bactericides, fungicides, steroid anabolic growth promoters and peptide production enhancers, Anti-parasite drugs (Abamectin, Albendazole, Azaperon, Doramectin, Ivermectin, and Fenbendazole), Antibiotics (Penicillin, Tetracycline), amino-glycosides, and other food animal drugs (Delgado et al., 1999).

Some drugs are prohibited from use in food animals. Drugs that are not permitted to give food producing animals are: clenbuterol, chloramphenicol, nitroimdazoles, and nitrofurans. Chloramphenicol not used for any purpose that would result in the presence of residues in food animals for consumption by human beings. In veterinary medicine, the drug is approved for use only in the non-food producing animals (Mercer, 1975).

\subsection{Major Drugs Used in Beef Cattle}

There are many drugs used in beef cattle but, major ones are: penicillin, tetracycline, dihydrostreptomycin, macrolides, hormones and anti-helminthic. Penicillin derivatives ( $\beta$-lactam antibiotics) are widely used in beef cattle to treat infections and as feed or drinking water additives to prevent some diseases. Preparation containing a combination of benzathine penicillin, procaine penicillin, and dihydrostreptomycin are used in mixed to synergize their action on pathogens (Mercer et al., 1970).

Oxytetracycline is a broad spectrum antibiotic used to treat a variety of infections and is also used as a growth promoter in animals. Addition of tetracycline to animal feeds at 5-20ppm does not seem to produce residues in edible tissues. In addition to these; neomycin, gentamicin, flunixin, streptomycin, erythromycin, antihelminthic, and other drugs are also used in beef cattle (Commission of the European Communities, 2005).

Many feed additives are available that can improve rate of gain and feed efficiency or prevent certain diseases. Feed additives may be both nutritive and non-nutritive; and work by either direct or indirect methods on the animal's system. Many of the products influence different systems. Therefore, the effects of one can be additive to another. Since some feed additives must be withdrawn from the diet before slaughter, the beef cattle feeder has crucial responsibilities. The use of medicated feed additives is controlled by the Food and Drug Administration (FDA). Other feed additives such as larvacides are controlled by the Environmental Protection Agency (EPA) since they are not absorbed and have no direct physiological impact on the animal (Tishler et al., 1990).

Growth promotants are primarily given to cattle in the form of small pelletsplaced under the skin of the animal's ear. These ear implants dissolve slowly over a 100-120day period. The ear is used to inject, because ears do not enter in the food supply. The active ingredients most of which are naturally occurring hormones are either oestrogens or androgens. The oestrogens can be natural, synthetic or plant based. The androgens also may be either natural or synthetic. The synthetic androgen used in implants (trenbolone acetate) has low adverse effect on public health (Redundant and Moreitain, 1990). Depending on the implant, age and sex of the animal, implants will improve growth rate from 10 to 20 percent and decrease the cost of beef productionby 5 to 10 percent (Sbampato et al., 2001). 


\subsection{Drugs Used in Dairy Cattle}

The most commonly used drugs in dairy cattle are: penicillin, tetracycline, amino-glycosides, ceftiofur, chlorhexidine, povidone iodine, polymixin B, macrolides, Sulfonamides and other drugs. Dairy cattle that have been treated with antibiotics produce milk containing antibiotic residues for a period of time after treatment. Treated cows are therefore required to be excluded from the milk supply for a specific time period to ensure that antibiotic residues no longer remain in their milk (Mcevoy et al., 1999).

Extra label intramammary administration of drugs occurs with some regularity in dairy cattle most commonly in association with mammary gland infections (mastitis) that fail to respond to approved products (Smith et al., 2004).

Ceftiofur sodium and ceftiofur hydrochloride are approved for intramuscular and subcutaneous use only and label use does not result in drug concentrations in milk greater than the tolerance limit level. Occasionally, these antimicrobials have been used in an extra labeled manner by bovine practioners or dairy producers for the inframammary treatment of coliform mastitis (Smith et al., 2004).

Chlorhexidine solution is occasionally, used to cause cessation of lactation in chronically infected mammary gland quarters. After its intramammary infusion, no residues were detected in milk from untreated mammary gland quarters. However, it was detected in milk from treated quarters throughout the duration (Middleton et al., 2003).

Polymixin B is narrow spectrum antimicrobial with good in-vitro activity against gram negative bacteria including coliforms after intramammary infusion. The drug was not systemically absorbed from normal udders although: substantial blood and urine polymixin concentrations were detected after infusion of acutely inflamed udder (Ziv and Schulze, 1982).

Povidone iodine is also given by the intra-mammary route for cessation of lactation in chronically infected mammary gland quarters. It may be less likely to cause milk residues than chlorhexidine (WHO, 1963).

Because povidone iodine is very effective completely eliminating all secretions from treated mammary gland quarter, it is likely a better choice for therapeutic cessation of lactation because the residues risk should be minimal if no secretion (milk) is produced. However, it should be emphasized that this represents extra label use of drug and because appropriate milk withdrawal data are not available extra care must be taken to avoid milking treated mammary gland quarters, which could potentially lead to residues (Middleton and Fox, 2001).

\subsection{Drugs Used in Poultry}

Antibiotics are used on a large scale in poultry farms to treat or prevent diseases to promote growth (Richard, 1986).

Many antibacterial and anticoccidial drugs are licensed for use in poultry for treatment of enteric and respiratory disease. Veterinary drugs are therapeutically used for laying hens, generally by mass application via water or feed or may reach them accidentally: for example, as a result of cross contamination in the feed mill (WHO, 1963). The major drugs used in poultry are: chlortetracycline, sulfonamides, erythromycin, gentamicin, furazolidone, nitrofurazole, tylosine, lincomycin, ampicillin, doxycycline, neomycin and other drugs are used for the treatment and prophylactic purposes. Sulfonamides in poultry are widely used for the treatment of infectious corynza, pullorum disease, fowl typhoid, and coccidiosis The compounds roxarsone, carbarsone, and arsanilic acid have been used in drugs added to feed for chickens, and turkeys to prevent disease, increase feed efficiency, and promote growth (Giguere et al., 2006).

\section{Drug residues in edible tissues}

The drugs or their metabolites left over in the body after their administration for a long time are termed as residues. After the treatment of infected animals with drugs, the residues of drugs are present at some level in edible products like milk, eggs, and meat of treated animals. Drug residues concentrations vary considerately from tissue to tissue and are generally observed to be higher in tissues of storage such as body fat or in organs that actively metabolize and excrete them. For example, the residue may be highest in body fat, in the liver and kidneys (Booth, 1973).

Chlorinated compounds such as pesticide agents have a high affinity for storage in the fat. Internationally, the levels of antibiotics that may be added to feeds without veterinary medical prescription vary from country to country. In general, use of antibiotics that might result in deposition of residue on meat, milk, and egg must not be permitted in food intended for human consumption. However, if the use of antibiotics is necessary as in the treatment of animal diseases, a withholding period must be observed until the residues are negligible or can no longer be detected (Rasmussen, 1998).

Concerns have been reflected about the presence of residues in food products and questions have arisen among various public health authorities about the wisdom of using low or sub therapeutic levels of antibiotics in the feed of animals. It is generally accepted that such levels have less than an optimum antimicrobial effect and that the induction of bacterial resistance including transference of R-factor can occur. There is sufficient 
evidence to indicate that heavily exposed human personnel have a higher incidence may be the result of exposure to antibiotics or antibiotic feed animals or their food products. However, the animals that serve as shedders of antibiotic resistance pathogens for general human population remain undetermined (Smith, 1974b).

\subsection{Drug Residues in Meat}

Drugs most likely to be detected in meat are: penicillin (including ampicillin), tetracycline (including chlortetracycline, and oxytetracycline), neomycin, gentamicin, streptomycin, flunixin, arsenicals, and sulfonamides (including sulfadimethoxine and sulfamethazine and sulfamethoxazole). Penicillin and sulfonamide drugs were most commonly detected at violative levels in swine and cattle. Neomycin and gentamicin were also detected in a number of cattle particularly, calves. Other drugs detected in cattle and swine included tilmicosin, flunixin, and tetracycline (Gregory, 1988).

According to Garrod (1964), antibiotics used in dry feeds at less than 20 ppm will not give rise to detectable levels in meat even if administration was continued until the time of slaughter. Under certain circumstances the WHO Expert Committee in 1963, acknowledged that it might be necessary to exceed this level. All edible tissues (liver, muscle, fat, and kidney) were found to be free from penicillin residue after 0.5 and 7 days of withdrawal respectively. In calves, penicillin residues were present in the injection site and in urine 45 days after treatment with a preparation containing a combination of benzathine penicillin, procaine penicillin and dihydrostreptomycin (Righter, 1971b).

Sulfonamides are generally used to treat a wide variety of bacterial and coccidial infections in food producing animals especially, in poultry and are used as growth promoters in swine. Consequently, drug manufacturers were not required to submit data on tissues residues following their use in food producing animals (Tishler et al., 1990).

In sheep, 10-daywithdrawal period is required for sulfamerazine when given at the recommended therapeutic level of $132 \mathrm{mg} / \mathrm{kg}$ for 3days. Most of analytical methodology for the detection of sulfonamide residues in tissues is sensitive to $0.1 \mathrm{ppm}$. For blood and serum, sensitivity to $2 \mathrm{ppm}$ has been reported (Righter et al., 1972).

Tetracycline was presented in the bones of chickens that were fed only $5 \mathrm{ppm}$ for no longer than 3 days. It has also been detected in bones at the level of $5.5 \mathrm{ppm}$ in chickens fed $9.2 \mathrm{ppm}$ for approximately 2 months, 0.52 $\mathrm{ppm}$ in swine fed $30 \mathrm{mg}$ /day for 3 months and $1.79 \mathrm{ppm}$ in calves fed $60 \mathrm{mg} /$ days for 2 months. The tetracycline absorbed from the gastrointestinal tract and may become firmly bound in teeth and skeletal structures (WHO, $1969 \mathrm{~b}$ ). In swine, the continuous feeding of tetracycline $100 \mathrm{~g} /$ ton in combination with penicillin $50 \mathrm{~g} / \mathrm{ton}$ and sulfamethazine $100 \mathrm{~g} /$ ton for 14 weeks resulted in less than $1 \mathrm{ppm}$ for chlortetracycline in all tissues on the day 0 of withdrawal (Babapour, et al., 2012).

The kidney contained 20 times the concentrations $235-288 \mu \mathrm{g}$ of dihydrostreptomycin/g of tissue of antibiotic compared to that detected in muscle $1.2-6 \mu \mathrm{g} / \mathrm{g}$ from the injection site or in the liver $14-21 \mu \mathrm{g} / \mathrm{g}$. In pigs treated for 3 days with dihydrostreptomycin at a dosage of $26 \mathrm{mg} / \mathrm{kg} /$ day in combination with penicillin, dihydrostreptomycin residues were detected for 60 days in the kidneys and muscle injection sites. In as much as the urine did not contain detectable dihydrostreptomycin at 60 days, an affinity, or binding capability of the antibiotic to renal tissue was suspected (Mercer et al., 1970).

\subsection{Drug Residues in Milk}

Antibiotics are invariably administered to dairy cattle to control infectious diseases, but their indiscriminate use, without adequate technical and veterinary control, can lead to a series of negative consequences at all levels of the dairy productive chain (Portugal, 2001). Antibiotic residues in milk and milk products are a rare consequence of antibiotic usage on dairy farms. The use of intra-mammary antibiotics and mistakes regarding withholding periods of milk are the most frequently cited reasons for antibiotic residues. Antibiotic residues enter the milk supply when treated cows are returned to the milking herd early or when a cow retains antibiotic residues in her system for an extra ordinary length of time (Henningson et al., 1963).

Probably the most common way milk is contaminated by penicillin is through intramammary infusion of the drug in the treatment of bovine mastitis. However, the administration of penicillin by other routes can also result in contamination of milk. The allergenic effects in human beings from the consumption of milk containing penicillin and other antibiotic residues were reported. The depletion of aqueous penicillin 100,000unit/quarter in the milk of dairy cattle requires 48 hours and the same dosage in oil requires 72 hours (Peoples and Pier, 1960).

In the fermented dairy products manufacturing, such as cheeses and yogurts, the presence of antibiotics and other antimicrobial agents can lead to the partial or total inhibition of the lactic bacteria resulting in products with compromised structural and sensory features (Kecelli and Robinson, 1997).

In the typical case of stretched curd cheeses, such as provolone and mozzarella, the stretching process becomes impossible and the fermentation periods can be very extensive. When the cheese curd is cut, it results in higher losses of casein and fat to the whey leading to yield reduction. Cheese curing is also affected due to the 
lack of lactic bacteria in the desirable biochemical reactions, such as proteolysis (Tronco, 2001).

Dairy processors can protect the milk supply by testing milk for penicillin, sulfa drugs, and other antibiotics before accepting each shipment at the dairy plant. In addition, efforts continue to educate producers and veterinarians on the need to eliminate drug residues from the milk supply. Processors have a key role in ensuring that milk containing drug residues is not accepted at dairy plants and processed into finished dairy products (Fellig and Westheimer, 1990).

\subsection{Drug Residues in Egg}

When therapeutically used veterinary drugs or their metabolites reach the blood stream of hens, they are distributed over the whole body, especially the ovary with growing follicles and the oviduct, where the egg white is formed and secreted (Radu et al., 2001).

Therefore, may increase the incidence of unacceptable residues in eggs. Such residues are reduced by establishing and adhering to withdrawal periods before slaughter and by sometimes prohibiting the use of certain antibiotics in laying hens (Kan and Petz, 2000).

Due to lack of biosecurity measures prevalence of infectious diseases and subsequently indiscriminate drug usage, without observing withdrawal period, has made the poultry products unsafe for human health (Weiss et al., 2007).

Sulfonamide drugs are most commonly used on poultry farms. These drugs can be easily absorbed and distributed through the body of the chicken, accumulate in various tissues and transferred into their products. The recommended withdrawal periods if not observed before slaughtering of the medicated animals and its products obtained from such animals may be contaminated with residues of sulfonamides (Weiss et al., 2007).

As a result, there is a concern that the residues may be retained in the meat and eggs that present a potential risk to human health. In order to decrease the potential risk to the consumer's health and to ensure the reduction of sulfonamide residues in edible tissues and eggs to an acceptable level, these substances must be administered only in recommended concentrations and their respective withdrawal times must be observed. The maximum residue level (MRL) of sulfonamides in poultry tissues and eggs is $100 \mu \mathrm{g} / \mathrm{kg}$ (Kozarova et al., 2004).

The pharmacokinetics of drug residues in yolk and egg white show certain common features. Residues of drugs appear first in egg white, where they reflect plasma levels. The time required to achieve constant levels in plasma and hence white is generally 2-3 days. Residues in yolk reflect the plasma levels during 10 days of their rapid growth, thus depending on the length and timing of the exposure relative to yolk growth, levels in yolk can increase, be constant or decrease (Tishler et al., 1990).

An exposure of about 8-10 days is necessary for yolk levels to become constant. A single exposure towards a drug might be sufficient to detect the drug in either egg white or yolk, depending on the characteristics of the drug and the sensitivity of the analytical method used for testing. Disappearance of drugs from white and yolk depends heavily on the plasma levels of the drug tested. Drugs that clear rapidly from the body also disappear from egg white in about 2-3 days after cessation of exposure (Franco et al., 1990).

Disappearance of drugs from yolk generally takes about 10 days. By that time, drug-containing lipoproteins taken up during the rapid growth phase of the yolk have been excreted with the eggs. However, if the exposure level is very high and the detection limit for the drug tested very low, residues deposited in the yolks, which are in the intermediate stage of growth, will also be detectable. This can explain the fact, chloramphenicol residues remain in eggs until 70 days after administration. The main compartment of eggs to be taken into account when considering drug residues has been disputed. It appears that the extent of exposure, as well as the characteristics of the drugs themselves is the determinant factors (Roudaut, 1998).

\section{Potential adverse effects of drug residues in human}

The presence of antibiotics in human food is associated with several adverse public health effects including allergic reaction, hypersensitivity, tissue damage, gastrointestinal disturbance, carcinogenic effect, and neurological disorders. Antibiotic resistance has become a definite complicating factor in the treatment of animal diseases. There is also concern that resistant organisms shed by animals may transfer R-factors to the enteric organisms of human beings and conceivably complicate the treatment of human disease (Aronson, 1975).

Although some hypersensitive effects reported in human beings have been associated with drinking milk containing antibiotic residues such as penicillin, is generally recognized that there are no known deleterious effects on human health attributable to drug residues permitted in food supply (Storrs et al., 1970a). An antibiotic residue in milk leads to: severe allergic reactions in sensitive consumers, culture failure, and subsequent loss of product, a change in consumer perception of milk being pure, unadulterated, natural product (Kozarova et al., 2002).

\subsection{Allergenic effect}

Allergic reaction to the human body mostly resulted from consumption of animal products especially milk that 
contaminated with $\beta$-Lactam antibiotics (DeWeck, 1983). The occurrence of antibiotic residues in milk intended for human consumption is undesirable for a number of reasons. Recently, the presence of antibiotics in milk has been prohibited because of concerns about public health. Initially, public health officials desired to protect hypersensitive individuals from exposure to specific antibiotics. Attention has shifted to the potential for antibiotic residues in milk to contribute to the development and/or transmission of antibiotic resistant bacteria (Mitchell, et al., 1998).

Anaphylactic reactions have been reported to result from consumption of beef or pork containing penicillin. Allergic reactions to antibiotics are well recognized and hypersensitivity to $\beta$-lactam compounds is especially prevalent (Kurwijila et al., 2006).

The immunological characteristics of most other drug classes (including macrolides, tetracycline, and amino-glycosides) make the development of allergic responses to minute residues. Unlikely, although it is considered theoretically, possible that exposure could result in clinically relevant immunological events. Allergic reactions (dermatitis, pruritis, and urticaria) of pre-sensitized individuals caused by $\beta$-lactam residues in milk have been documented for a small number of people (DeWeck, 1983).

Allergic effect of macrolide antibiotics (erythromycin, oleandomycin, and spiromycin) has not been observed in human beings following their clinical use. Residue level of erythromycin in milk, meat and egg as high as $0.04,0.3$ and $0.3 \mathrm{ppm}$ respectively; oleandomycin as high as $0.15,0.3$ and $0.1 \mathrm{ppm}$ respectively and spiromycin as high as $0.025 \mathrm{ppm}$ reported only in meat are not likely to cause allergic effect. Ingestion of antibiotics present in milk can influence gut flora (Sutiak et al., 2000).

\subsection{Microbiological effect}

Drugs can cause disruption of normal human flora in the intestine and bacteria that usually live in the intestine act as a barrier to prevent incoming pathogenic bacteria from getting established and causing disease. Antibiotic residue might reduce total numbers of these bacteria or selectively kill some important species (Woodward, 1991).

Bacteria that are sensitive to antimicrobials are killed or put at a competitive disadvantage, while bacteria that have the ability to resist antimicrobials have an advantage and are able to grow more rapidly than more susceptible bacteria. In addition, bacteria can become resistant when resistance genes are passed from a resistant bacterium to a sensitive one. Thus, antimicrobial agents may increase the prevalence of resistant bacteria among both target pathogens and normal bacterial flora (Gregory, 1988).

\subsection{Carcinogenic effect}

Drug whose residues induce cancer in humans or consumers are: Nitrofurans, Nitroimdazoles. Inorganic arsenic is also a known carcinogen and may adversely affect the circulatory and nervous systems. Although, furazolidone had been labeled and approved for anti-protozoal and other uses for a wide variety of conditions in poultry and swine, its metabolites also have been shown to induce cancer in animals and human beings (Gregory, 1988).

\subsection{Toxic effect}

Toxic effects mostly resulted from the consumption of food animal products medicated with drugs like: clenbuterol (Gustavsson et al., 2004). Toxic effects to human beings include hearing problems caused by streptomycin and allergy problems and anaphylactic shock caused by penicillin. In addition, it is widely recognized that the indiscriminate use of such substances promotes the development of resistant microorganisms hindering the antibiotic therapy. They are also heat resistant, so the temperatures used in the UHT process are ineffective in eliminating them (Brancher and Fagundes, 1998).

In tetracycline, some toxic effects were observed at high doses. Therapeutic doses are occasionally associated with discolored teeth, allergic reactions, or peripheral blood changes (Gustavsson et al., 2004).

Some antibiotics like streptomycin, neomycin and, gentamicin has been reported to cause damage to the kidney and to hearing. They depleted rapidly from muscle and fat but tend to persist in kidney and liver, not readily metabolized in animals or in humans (Eberhart et al., 1998).

One significant adverse effect of gentamycin in humans that occurred during treatment of pregnant women with tuberculosis and also infants of women treated first trimester: damage to a cranial nerve and congenital deafness. No other evidence of effects on fertility or reproduction. It is not expected that low food residues/low absorption would affect fetal development (Seguin et al., 1974).

Data cited by JECFA indicate that the primary mechanism of toxicity of sulfonamides is associated with the thyroid-hypothalamus. Toxicity should be measured by parameters of thyroid and pituitary function. Hypersensitivity reactions or primarily skin rashes to therapeutic levels of sulfonamides have been reported: but, there have been no cases that involved exposure to residues in foods (Fechner et al., 1974). 


\section{Conclusion}

Antibiotics are natural products of a micro-organism used for therapeutic, prophylactic, and nutritive and growth purposes in food animals. Drugs that used in food animals are Topical antiseptics, bactericides, and fungicides, Steroid anabolic growth promoters and peptide production enhancers, Anti-helminthic drugs, Antibiotics (Penicillin, Tetracycline), Amino-glycosides and others. Drugs most likely to be detected in meat are: neomycin, gentamicin, streptomycin, flunixin, arsenicals, penicillin tetracycline, and sulfonamides. Antibiotics are invariably administered to dairy cattle, but their indiscriminate use, without adequate technical and veterinary control, can lead to a series of negative consequences at all levels of the dairy productive chain. Many antibacterial and anticoccidial drugs are licensed for use in poultry for treatment of enteric and respiratory disease. Potential adverse effects of drugs that appear as residues in food animals are: carcinogenic, allergic, toxic, neurologic disorder and microbiological effects.

\section{References}

Aronson, A.L. (1975): Potential impact of use of antimicrobial drugs in farm animals on public health. Presented at the meeting on Pharmacology in the animal health sector. Colorado State University.

Babapour, A.,Azami, L., and Fartashmehr, J. (2012): Overview of Antibiotic Residues in Beef andMutton in Ardebil, North West of Iran: World Applied Sciences Journal 19 (10): 1417-1422.

Benet, J.J.,and Belleemain, V. (2005): Responding to consumer demands for safe food: A Major Role for veterinarians in the $21^{\text {st }}$ Century. Minneapolis: World Veterinary Congress.

Booth, N.H. (1973): Development of a regulatory research program in Veterinary Medical Toxicology: Journal of veterinary toxicology, 15: 100-100.

Brancher, C.,and Fagundes, C. M. (1998): Adaptation of Reductase Method to detect antibiotic residues in milk. Brazilian Journal of Agro science, 2(1): 80-84.

Commission of the European Communities (2005): Report on the results of residue monitoring in food of animal origin in the Member States.

DeWeck, A. L. (1983): Penicillin and cephalosporin. P. 423-482.In A. L. de Weck and H. Bundgaard (ed.),Handbook of experimental pharmacology, Vol.63. Springer-verlag KG, Berlin.

Delgado, C., Rosegrant, M., Steinfeld, H., Ehui, S., and Courbois, C. (1999): Food, Agriculture, and the Environment discussion paper 28. Washington, D.C:

Eberhart, R.J., Watros, G.H., Hokanson, J.F. and Burch, G.E. (1998): Persistence of Antimicrobial agents in milk after intramammary treatment of clinical mastitis. Journal of American Veterinary MedicineAssociation, 143:390.

Fechner, T., Topfer, H., and Pannwitz, E. (1974): BeitragzurRuckstands-analtika und dynamic Von

Fellig, J., and Westheimer T. (1990): Determinations of sulfadimethoxine in animal tissues. Journal Agriculture Food chemistry, 4:13-16.

Franco, D.A., Webb, J., and Taylor, C.E.(1990): Antibiotic and sulphonamide residues in poultry meat: Implication for human health. Journal of Food Protein 53:178-185.

Garrod, L.P. (1964): Source and Hazards to man of antibiotics in foods. Proceeding Research Society Medicine57: 1087

Giguere, S.J., Prescott, J.D., Baggot, R. D., Walkerand. P., and Dowling,M. (2006): Antimicrobial therapy in veterinary medicine. $4^{\text {th }} e d$. Blackwell Publishing Ltd, Oxford, UK.

Gregory, M. (1988): Food Toxicology. Pp1-12. University of Idaho.

Gustavsson, E., Degelaen, J., Bjurling, P., and Sternesjö, A. (2004): Determination of beta lactams in milk using a surface plasmon resonance-based biosensor.

Henningson, R.W., Hurst, Moore, S.L., and Kelly, J.W. (1963): Effect of intra uterine infusion of penicillin streptomycin and furacin and vaginal deposition of furacin on chemical residual levels in milk. Journal Dairy science, 46:195.

Jones, G.M and Seymour E.H. (1988): Cow side antibiotic residue testing. Journal of Dairy Science, 71(6): 1691.

Kan, C.A., and Petz, M. (2000): Residues of veterinary drugs in eggs and their distribution between yolk and white. Symposium on Agrochemical residues in eggs, American Chemical Society.

Kecelli, T. and Robinson, R. K. (1997): Dairy products the real risk. Dairy Industries International, 62(1): 29-33

Kozarova, I., D., Mate, R. Cabadaj, C., and Hussein, K. (2002): Methods for Determining Sulfonamide residues in meat. In Proceedings of Lectures and Posters of the InternationalConference.HygienaAlimentorum. XXIII.Strbske Pleso. High Tetras.SR,65-67.

Kozarova, I., D., Mate, K. Hussein, K., Raschmanova, Marcincak, S., and Jevinova, P. (2004): HighPerformance Liquid Chromatographic Determination of Sulfadimidine Residues in Eggs. Acta Veterinaria, 54(5): 427-435.

Kurwijila, L.R., Omore, A., Staal, S., and Mdoe, N.S. (2006): Investigation of the risk of exposure to antimicrobial residues present in marketed milk in Tanzania. Journal of Food Protection, 69(10): 487-2492. 
Lee, M.H., Lee, H.J., and Ryo, P.D. (2005): Public health risks: chemical and antibiotic residues review. AsianAustralasian Journal of Animal Sciences, 14: 402-413.

Mcevoy, J. D., Mayne, H.C., Higgins, and Kennedy, D.G. (1999): Transfer of sulphamethazine from contaminated dairy feed to cows' milk. Veterinary Record 144: 470-475.

Mercer, H.D. (1975): Antimicrobial drugs in food producing animals. Journal of Veterinary Clinical in Northern America, 5: 3-18.

Mercer, H.D., Rollins, L.D., Garth, M.A., and Carter, G.G. (1970): A residues study and composition of penicillin and dihydrostreptomycin concentrations after intramuscular and subcutaneous administration in cattle. Journal of American Veterinary Medicine Association, 158: 776.

Middleton, J.R., Hebert, V.R., and Fox, L.K. (2003): elimination kinetics of chlorhexidine in milk following intra-mammary infusion to stop lactation in mastitis mammary gland quarters of cows. Journal ofAmerican Veterinary Medicine Association,222:1746-1749.

Middleton, J.R., and Fox, L.K. (2001): Therapeutic cessation of lactation of staphylococcus aureus infected mammary gland quarters. Journal of Dairy science, 84:1976-1978.

Mitchell, J.M., Griffiths, M.W., McEwen, S.A., McNab, W.B., and Yee, A.J. (1998): Antimicrobial drug residues in Milk and Meat: Causes, Concerns, Prevalence, Regulations, Tests, and Test performance. Journal of Food Protection, 61: 742-756.

Moat, W.A. (1988): Inactivation of antibiotics by heating in foods and other substrate-a review. Journal of Food Protocol. 51: 491-497.

Peoples, S.A., and Pier, A. C. (1960): Drug levels in milk. Module of Veterinary practice41:26.

Portugal, J.A. (2001): Quality of milk and antibiotic residues. Revista do Instituto de Laticínios Cândido Tostes, 56(309): 9-14.

Radu, S., Mutalib, S.A., Rusul.G, Hassan, Z., and Yeang, L.K. (2001): Molecular Characterization of Salmonella weltevreden isolated from poultry: Evidence of Conjugal Transfer of Plasmid and Antibiotic Resistance Microbes, 104(407): 39-47.

Rasmussen, F. (1998): Mammary excretion of sulfonamides. Acta pharmacol Toxicological, 15:139.

Redundant, B., and Moreitain, J.P. (1990): Sulphonamide residues in milk of dairy cows following intravenous injection. Food Additives and Contaminants, 7:527-533.

Richard A.H., and Clarsonce, M.F. (1986): The Merck veterinary manual 6 ed., Merck Co., Inch, U.S.A.

Righter, H.F., (1971b): Tissue residue depletion of sulfathiazole in swine. Journal of Animal science33: 797.

Righter, H.F., Worthington, J.M., Zimmerman, H.E., and Mercer, H.D. (1970): Tissue Residue depletion of sulfaquinoxaline in poultry. American Journal of Veterinary Research,31:1051.

Righter, H.F., Worthington, J.M., and Mercer, H.D. (1972): Tissue residue depletion of sulfamerazine in sheep. Journal of Agriculture and Food Chemistery, 20:876.

Roudaut, B. (1998): Elimination of oxolinic acid in eggs after oral treatment.

Sbampato, C.G., Abreu, L.R., and Mendonça, A.T. (2001): Technological Aspects of yogurt and cheese processing using milk with antibiotic residues. Revista do Instituto de Laticínios Candido Tostes. 54(313):3-19.

Smith, G.W., Gehering, R., and Riviere, J.E. (2004): Elimination kinetics of ceftiofur hydrochloride after intramammary administration in lactating dairy cows. Journal of American Veterinary Medicine Association, 224: $1827-1830$.

Smith, H.W. (1974b): Antibiotic resistance bacteria in animals: The dangers to human health. Breeding VeterinaryJournal, 130:110.

Storrs, B., Thompson, J., Fair, G., Dickerson, M.S., Nicky, L., Barthel, W., and Spauding, J.E. (1970a): Organic mercury poisoning. Morb Mortal 19:25.

Sutiak, V.I., Korenek, M., Krokavec, M., Kozak, J., Saly, and Neuschl, J. (2000): Current problems with drug use and the need for their solution in poultry and some other animals. In Proceedings of Lectures and Posters of the International Conference. Hygiena Alimentorum. XXI: Pp113-115.

Tishler, F., Sutter, J.L., Batish, J.N., and Hangman, H.E. (1990): Improved method for determinations of sulfonamides in milk and tissues. Journal of Agriculture and FoodChemistry 16:50.

Tronco, V. M. (2001): Qualidade Na Inspeção Do Leite. Rio Grande do Sul: Editora UFSM, 234. Vet Res 31: 1589.

Wassenaar, T. M. (2005): The use of antimicrobial agents in veterinary medicine and implications for human health. Critical Reviews in Microbiology, 31: 155-169.

Weiss, C.A., Conte, C., Milandri, G., Scortichini, P., Semprini, R., Usberti, and G. Migliorati. (2007): Veterinary drugs residue monitoring in Italian poultry: current Strategies and possible developments. Food Control. 18: 1068-1076.

WHO (1963): The public health aspects of the use of antibiotics in food and feed stuffs. WHO Technical Report series, 260. 
WHO (1969b): Specifications for identify and purity of food additives and their toxicological evaluation: some antibiotics. World health organization Technical Report series, 430

Woodward, K.N., (1991): Hypersensitivity in humans and exposure to veterinary drugs. Veterinary and Human Toxicology; 33: 168-172

Ziv, G. and Schulze, W.D. (1982): Pharmacokinetics of Polymixin B administered via the bovine mammary gland. Journal of Veterinary pharmacology andTherapy, 5: 123-129. 